\title{
Agrupamientos escolares y retos para la educación inclusiva en infantil y primaria
}

\author{
School groupings and challenges for inclusive education in early childhood and \\ primary education
}

\author{
Pablo Cortés González \\ pcortes@uma.es \\ Universidad de Málaga. España \\ Blas González Alba \\ blasbatera@hotmail.com \\ Consejería de Educación. Junta de Andalucía. España \\ María de Fátima Sánchez Mesa \\ fatimasanchezmesa@gmail.com \\ Universidad de Málaga. España
}

\section{Resumen}

En este estudio, por un lado, queremos comparar los agrupamientos escolares que se desarrollan en dos centros educativos de Educación Infantil y Primaria de la provincia de Málaga y, por otro lado, observar el impacto que genera en la organización del aula la implantación de un proyecto de innovación educativa que busca generar una perspectiva inclusiva en las prácticas docentes. Se han utilizado entrevistas semiestructuradas, cuestionarios, observación, revisión de informes finales del proyecto y grupos de reflexión. Los resultados en ambos centros han sido similares, encontrando cambios metodológicos más significativos en los grupos de Educación Infantil. Concluimos aportando retos para agrupar al alumnado en el aula desde una perspectiva inclusiva.

Palabras clave: Agrupamientos escolares; organización del aula; innovación; inclusión educativa.

\section{Abstract}

In this study, on the one hand, we want to compare the school groupings that are developed in two schools of Early Childhood and Primary Education in the province of Malaga and, on the other hand, to observe the impact generated in the organization of the classroom by the implementation of a educational innovation project that seeks to generate an inclusive perspective in teaching practices. Semi-structured interviews, questionnaires, observation, review of final project reports and reflection groups have been used. The results in both centers have been similar, finding more significant methodological changes in the Early Childhood Education groups. We conclude by providing challenges of groupings in the classroom from an inclusive perspective.

Keywords: School groupings; classroom organization; innovation; inclusive education.

Cómo referenciar este artículo / How to reference this article:

Cortés, P., González, B., \& Sánchez, M.F. (2018). Agrupamientos escolares y retos para la educación inclusiva en infantil y primaria. Tendencias Pedagógicas, 32, inicial-final. doi: 10.15366/ tp2018.32.006 


\section{Introducción}

El aula representa, desde un punto de vista formal de la educación, el lugar donde se desarrollan principalmente los procesos de enseñanza-aprendizaje, entendiendo que, en ocasiones, se incluye en su definición otros espacios que puntualmente la sustituyen; como, por ejemplo, la biblioteca del centro, las pistas deportivas, el aula de plástica, etc. Se contempla, desde una mirada organizativa, que la mayoría de las escuelas siguen manteniendo este espacio como lugar de referencia, convirtiendo al aula en el espacio donde el alumnado pasa la gran parte de las horas lectivas.

Su organización, uso o aspecto son factores íntimamente relacionados con el perfil o identidad del docente; sin perder de vista el tipo de proyecto educativo del centro. Esto último alude a una visión más amplia de entender la organización del aula. En cierta medida, las múltiples formas que van a determinar su organización se circunscriben a prácticas que pueden ser de carácter más o menos tradicional o innovador y que se amparan en las diferentes concepciones que tienen los docentes de entender la enseñanza, de las que destacamos dos visiones amplias: (1) como práctica para la adquisición de conocimientos o (2) como práctica educativa y emancipadora (Murillo, Martínez y Hernández, 2011). No obstante, de acuerdo a lo planteado, en la escuela podemos encontrar "tantas aulas distintas como pedagogías diferentes deban ponerse en práctica en su interior" (Trilla y Puig, 2003, 52), las cuales van a configurar las relaciones y los procesos de enseñanza-aprendizaje que en ella se desarrollen.

Es este punto el que nos motiva para la realización de la investigación educativa que presentamos en este artículo denominada 'ReIO - Resiliencia, Inclusión y Organización del Aula', de la que mostramos los primeros resultados obtenidos sobre la organización del aula en dos centros rurales de la provincia de Málaga.

Debemos mencionar que la presente investigación incluye entre sus estrategias empíricas, el proyecto de innovación educativa en centros escolares "Emprende tu vida" (PIN-091/16), aprobado por la Junta de Andalucía. Este proyecto se desarrolla con el objeto de promover prácticas educativas que favorezcan la inclusión escolar, el trabajo cooperativo y el desarrollo de las inteligencias múltiples (Gardner, 1983) y emocional (Goleman, 1996), a partir de estrategias educativas a implementar en el currículum escolar en las aulas de educación infantil, primaria y secundaria.

Para este artículo, se ha seleccionado la información recogida en dos de los tres centros que han participado en el proyecto de investigación. Las preguntas que nos planteamos abordar en este trabajo tienen que ver con dos cuestiones fundamentales: la primera, cómo han venido organizando los maestros y maestras los espacios escolares de infantil y primaria, desde el punto de vista de los agrupamientos de su alumnado en el aula, dependiendo de las necesidades y las materias a impartir; y, la segunda cuestión, qué cambios han realizado durante y después de haber participado en el mencionado proyecto de Innovación docente respecto a la perspectiva de la inclusión educativa y el trabajo cooperativo.

\section{Marco teórico}

Las distintas teorías de organización educativa entienden que la estructuración espacial y organizativa del aula es un factor clave en los procesos de aprendizaje (Laorden Gutiérrez y Pérez López, 2002), así como en los de socialización. Al respecto, podemos considerar el aula, primero, como un recurso didáctico (Visedo, 1991), ad hoc a los contenidos y a las metodologías, ya que de alguna manera interfiere directamente en qué tipo de aprendizajes y experiencias se desarrollan; y, segundo, como un espacio de participación ciudadana (dimensión política), en el que se conforma la cultura escolar y democrática del alumnado y del profesorado hasta el punto que "los propios aprendizajes que se producen, se entienden solo a la luz de los patrones establecidos por esa cultura organizativa" (Rivas, 2003, 111), de la cual los profesionales de la educación son los responsables y suelen guardar dependencia con las relaciones que se generan al respecto, sean del tipo que sean. 
En otras palabras, desde una mirada micro-política escolar (Ball, 1990), el aula se concibe como un lugar de interacción interpersonal, en el que aspectos como su organización y metodología van a configurar el proceso educativo, las relaciones sociales y educativas que en ella se produce; elementos que son percibidos y configurados por todos los agentes participantes en el aula (Ruiz Ruiz, 1994) y que van a determinar, en gran medida, la realidad socio-cultural y escolar de la misma.

$\mathrm{Al}$ respecto, Terrén (2004) plantea que la organización escolar se configura como un sistema de comunicación, donde la acción educativa se establece en torno a la "práctica de aula", y el aula se configura como un escenario en continua transformación en un nivel micro-micro (Ball, opus cit.). Añade, la necesidad de destacar los conflictos que trae consigo la micro-política escolar (organización escolar), por entender que:

"La cultura de la organización es un campo esencialmente conflictivo, en el que compiten o coexisten diferentes interpretaciones y manejos de la situación. La micropolítica de la interacción de redes supone, por tanto, una visión conflictiva de la vida cultural de la organización, que responde a un reconocimiento de su diversidad interna." (Terrén, 2004, 194)

La amplia diversidad de configuraciones intra-aula a las que alude Terrén, está condicionada, de acuerdo con Blase (2002), "a todos los tipos de estructuras y procesos para la toma de decisiones en los centros" y se manifiestan en el uso y gestión del espacio disponible, del tiempo, de los contenidos y de otros recursos escolares.

En este sentido, son recurrentes las aportaciones de Rivas cuando expresa que "la organización escolar es vista como un componente básico del proceso educativo" (2003, 111), y la estructuración y organización del aula representa una concreción de esa "organización escolar", que tiene que ver con el tipo de relaciones -pedagógicas y escolares- que se establecen entre los distintos agentes educativos: docentes, alumnado y familias (de manera más o menos directa).

Estas relaciones -entre las que se encuentran las prácticas escolares inclusivas- se plasman en el diálogo educativo y escolar que se establece entre los diversos actores (principalmente docentediscente) que van a configurar las dinámicas y los procesos de enseñanza-aprendizaje. Entendemos, por tanto, que las prácticas escolares se entienden en el marco de interacción interpersonal, independientemente de la perspectiva o paradigma en la que se sitúe la práctica docente.

No obstante, a modo de hipótesis, manifestamos en el proyecto que presentamos nuestro posicionamiento pedagógico al entender que las actuaciones inclusivas y cooperativas son las que van a favorecer prácticas escolares y educativas integradoras y significativas, poniendo el énfasis en la interacción, cuestión que venimos abordando en párrafos atrás. En este sentido, la educación inclusiva permite y ha permitido la evolución de nuestro sistema educativo a través de la creación de una escuela que educa teniendo en cuenta la diversidad como un factor inherente a la naturaleza y condición humana, y que la concibe como una fuente de enriquecimiento y de mejora que repercute en la calidad de la enseñanza y en la formación del alumnado.

Delgado la define como aquella que "implica que todos los niños y las niñas de una determinada comunidad aprendan juntos independientemente de sus condiciones personales, sociales $\mathrm{o}$ culturales, incluidos aquellos que presentan alguna condición especial que les dificulta el aprendizaje" $(2007,51)$. Esto significa, construir una escuela flexible, que tenga la capacidad de adaptarse para responder a las características de una población de jóvenes concreta, basada en la aceptación de la diversidad entendida como un fenómeno social, vivo, real y, por tanto, humano, es decir, una escuela de todos y para todos.

Otros autores, en investigaciones más recientes (Ainscow, Booth y Dyson, 2006), han ampliado la visión de la inclusión educativa, en la que han analizado otros modos de desarrollar unas prácticas educativas inclusivas teniendo en cuenta su propia realidad ecológica y adaptándose a las demandas y necesidades del alumnado. Esto nos constata, que, dentro de la perspectiva de la inclusión escolar, de acuerdo con Dyson (2010), no existe un único modelo de escuela inclusiva, entendiendo que lo 
que se pensaba como dificultad del aprendizaje, no es un dilema pedagógico en sí mismo, sino una cuestión de tradiciones escolares basados en el control y el poder. La inclusión desborda esta mirada deficitaria de entender a los sujetos.

Por lo tanto, la diversidad cultural, cognitiva y funcional deben entenderse como las disposiciones con las que cuenta el propio ser humano para su aprendizaje; es decir, son condiciones, que no limitaciones, para aprender; por ende, hay que entenderlas como oportunidades de aprendizaje.

Los agrupamientos escolares inclusivos tienen un papel fundamental en esto, tal y como demuestran recientes investigaciones (Rojas Tejada et al., 2012; Guasp, Ramón y De la Iglesia Mayol, 2016), pues materializan en la experiencia escolar lo que significa educarse en diversidad. Centrándonos en este concepto, vemos necesario aludir a autores como Morán, Molina y Siles (2012) que, basándose en los agrupamientos que propone el ministerio de Educación y Ciencia en el Plan Nacional de I+D (2008-2011), contemplan tres modalidades de agrupamientos escolares. Estas modalidades de agrupamiento escolar se asientan en los resultados obtenidos de la investigación INCLUD-ED, la cual propone los modelos Mixture, Streaming e Inclusivo para atender a la diversidad del alumnado bajo los principios de inclusión, normalización e integración educativa que la actual legislación contempla.

La primera modalidad, el modelo Mixture, hace referencia a la organización tradicional del aula; encontramos a un único docente que atiende a un grupo heterogéneo de alumnado en relación a su nivel de desarrollo competencial. En esta modalidad los objetivos, contenidos y actividades son las mismas para todos, lo que, pensamos, no permite atender de forma efectiva las características particulares del alumnado. Además, con la entrada del bilingüismo en las escuelas, esta primera modalidad organizativa hace aún más difícil la atención a la diversidad del alumnado.

La segunda modalidad, el modelo Streaming, clasifica al alumnado según su nivel de rendimiento escolar dentro de la misma aula o en otra; este sistema cuenta con la ayuda de otros docentes y categoriza al alumnado en función de sus características personales y competenciales. Desarrolla programas paralelos e itinerarios diferenciados en los que, a la postre, es la divergencia o la individualidad del alumnado lo que orienta la práctica escolar.

La tercera modalidad, el modelo de inclusión o inclusivo, permite atender la diversidad de todo el alumnado en el mismo aula y con la ayuda de otros docentes y colaboradores. Desde este modelo inclusivo la heterogeneidad es entendida como fuente de enriquecimiento en el que se reconocen las diferencias de las personas como aspecto fundamental para el aprendizaje.

Al hilo de esta tercera modalidad, que es donde nos situamos, distinguimos un siguiente nivel o paso en la perspectiva de inclusión educativa. Esta persigue una mirada educativa que, desde una perspectiva holística, pretende ser más justa, contextualizada y adaptada a las particularidades reales del alumnado a través de respuestas flexibles (García, 2013), y/o modificaciones en las metodologías de enseñanza-aprendizaje (Casanova, 2011), ambas relacionadas con estrategias de organización del aula. Esto es, incluir no solo una mirada organizativa, sino vincularla con una mirada metodológica.

Es decir, profundizar en esta mirada nos hace plantearnos retos en cuanto a la organización de los espacios escolares, del mobiliario, del aula y, por tanto, de las metodologías, enfocadas a ser elementos experienciales a disposición del alumnado para educar en diversidad. Es una cuestión de pensar estrategias que modulen la práctica educativa, de establecer objetivos (Segovia y Beltrán, 1998; Segovia Olmo, 2003), de acuerdo con la realidad cotidiana de la práctica docente. Es decir, le otorga a la organización escolar un carácter dinámico que permite su reestructuración en función de las actividades que en ella se vayan a desarrollar. 


\section{El proyecto de investigación}

\subsection{Antecedentes y contexto}

El aula, como hemos apuntado anteriormente, se convierte en el principal espacio de interacción escolar, así como el lugar en el que se desarrollan principalmente los procesos de enseñanzaaprendizaje, pues esta se ha instaurado como "el encuadre físico por excelencia diseñado para facilitar el tránsito de la cultura y el aprendizaje recíproco y vivencial de actitudes, normas y valores" (Trilla y Puig, 2003, 52), y así es entendido por docentes, familias y alumnado de un modo generalizado.

Lo que hace interrogarnos sobre este campo de estudio tiene fuertes vinculaciones con lo que hemos podido constatar en nuestra práctica docente, ya sea en el marco de la investigación y formación de maestros y maestras (Cortés, Leite y Rivas, 2014; Rivas et al., 2010), o en la práctica escolar como maestro de Pedagogía Terapéutica o como maestra de educación primaria; en este sentido, pensamos que la organización y los agrupamientos en el aula están cambiado ligeramente en los últimos años, a pesar de entender que la configuración del aula y la disposición alumnado influye de un modo significativo en los procesos de enseñanza-aprendizaje y en la inclusión educativa y escolar.

Esto nos ha conducido a plantear preguntas en torno a cómo los docentes optan por tipos de agrupamientos que se reiteran y/o modifican a lo largo de todo el curso en función de las diversas actividades que desarrollan, de su experiencia, competencias, estrategias de enseñanza-aprendizaje, asignaturas que imparten, objetivos que se proponen, etapa y curso educativo..., y cómo estos se configuran, o no, en prácticas de educación inclusiva. Consideramos que la organización del aula se refleja en el interés y aprendizaje del alumnado, debido a que, en función de la disposición de las mesas, por ejemplo, se propicia que se haga efectiva un tipo de metodología u otra.

La investigación consta de tres fases, no consecutivas sino complementarias, que detallamos a continuación:

- Fase diagnóstica. Estudio sobre la organización del aula, en el que a través de entrevistas, observaciones y cuestionarios al profesorado implicado desvelará qué tipo de agrupamientos y organización educativa desarrolla en sus aulas y en qué medida se consideran prácticas inclusivas.

- Fase reflexiva y formativa. Grupos de formación, de discusión y reflexión compartida a raíz de los datos rescatados y en el marco del proyecto de innovación docente señalado en la introducción.

- Fase propositiva. Propuestas de prácticas para la inclusión educativa y la generación de una red de maestros y maestras de la provincia de Málaga.

En la fase reflexiva y formativa toma especial relevancia, como apuntamos, el proyecto de innovación. Cabe mencionar que se ha desarrollado en uno de los centros educativos -el cual hemos llamado grupo experimental- una formación a lo largo del curso 2016/17 titulada "Estrategias Metodológicas para la Innovación en el aula" con el objeto de que los docentes incorporen a sus aulas prácticas, estrategias y metodologías educativas y escolares que requieran del uso de diversos agrupamientos con el fin de potenciar la inclusión y la participación de todo su alumnado. Esta experiencia la hemos recogido como estrategia empírica.

Para ofrecer más detalles, esta formación ha contado con muy diversas actividades: (1) se han realizado dos sesiones de formación que han tenido una duración de ocho horas presenciales distribuidas en dos sesiones de 4 horas que se han desarrollado en el primer y segundo trimestre respectivamente-; (2) otras dos sesiones de puesta en común -en gran grupo- en la que los docentes han expuesto sus percepciones, dificultades o cualquier otro aspecto acerca del desarrollo y/o implantación del proyecto; (3) la realización de tres actividades relacionadas con el proyecto -a 
través de la plataforma virtual Colabora 3.0 de la Junta de Andalucía-; y (4) la implantación en el aula de dos actividades que impliquen trabajo en pequeños grupos y gran grupo. Toda la formación desarrollada ha estado relacionada con metodologías cooperativas y participativas, la diversidad de agrupamientos, actividades para el desarrollo de las inteligencias múltiples y la inteligencia emocional.

Volviendo al contexto del proyecto de investigación, los escenarios los hemos vinculado con la temporalización del proyecto, y con el objeto de tener experiencias e información de una amplia variabilidad de contextos dentro de la provincia de Málaga. A continuación señalamos: primer año, zona rural; segundo año, zona urbana; tercer año, centros de compensatoria; cuarto año, zonas en transformación social; y quinto año, estudio comparativo y generación de comunidad de prácticas de maestros y maestras.

En referencia a los resultados que presentamos en este artículo, tienen que ver con la fase diagnóstica y reflexivo-formativa en dos centros educativos rurales participantes de la provincia de Málaga durante el curso 2016/17. Uno de ellos, ha actuado como grupo de control y el otro como grupo.

\subsection{Método}

\section{a) Objetivos}

La investigación se está desarrollando en torno a dos objetivos principales:

- Comparar los agrupamientos escolares que se desarrollan en centros de Educación Infantil y Primaria de la provincia de Málaga.

- Evaluar la incorporación de estrategias organizativas y metodológicas inclusivas del proyecto de innovación docente 'Emprende tu vida'.

- Así mismo, nos planteamos los siguientes objetivos específicos.

- Desarrollar formaciones sobre estrategias metodológicas asociadas a la innovación educativa y a la inclusión.

- Generar redes docentes para la reflexión y el aprendizaje mutuo.

- Analizar las prácticas organizativas del aula del profesorado participante.

- Analizar las prácticas inclusivas en el aula junto al profesorado participante.

\section{b) Participantes}

En relación a la muestra, se ha contado con dos centros de educación infantil y primaria. En uno de los centros (el que ha servido como experimental), uno de los autores trabaja como maestro de Pedagogía Terapéutica, lo que ha facilitado el acceso para todo el trabajo de campo y de análisis. La elección del centro educativo y de los docentes que han formado parte del grupo control se ha realizado teniendo en cuenta dos aspectos fundamentales: primero, por las condiciones contextuales y educativas en las que se ubica, debían ser similares al centro que ha actuado como experimental y, segundo, porque los docentes reunieran similares características de género, tutoría y/o especialidad y régimen laboral (situación de interinidad o destino en el centro).

Ambos centros se ubican en la provincia de Málaga, y tienen características contextuales, organizativas y educativas similares, pues, cuentan con dos líneas educativas, atienden a un número similar de alumnos (entre 350 y 400), son centros de educación infantil y primaria, están situados en poblaciones cercanas a grandes núcleos poblacionales $(10-15 \mathrm{Km}$.), y ambos pueblos tienen un nivel socioeconómico medio con familias de clase obrera y trabajadora que viven principalmente de la ganadería y/o de la agricultura.

Respecto a los sujetos participantes, han sido un total de 37 docentes de educación infantil y primaria; 17 han formado parte del grupo control (y por lo tanto de un centro educativo) y 20 del 
grupo experimental (del otro centro participante).

Sobre los docentes que imparten docencia en educación infantil cabe decir que el grupo control está formado por seis maestras de educación infantil (cuatro interinas y dos con destino definitivo en el centro), y del centro educativo que ha actuado como grupo experimental han participado seis maestras de educación infantil (tres interinas y tres con destino definitivo en el centro).

En la investigación han participado también 25 docentes de educación primaria, el grupo control se ha compuesto de cuatro maestros (uno interino y tres con destino definitivo en el centro), y siete maestras (tres interinas y cuatro con destino definitivo en el centro). El grupo experimental ha estado formado por seis maestros (dos interinos y cuatro con destino definitivo en el centro) y ocho maestras (tres interinas y cinco con destino definitivo en el centro). De los 25 docentes de Educación Primaria entrevistados 23 de ellos han sido tutores y especialistas y dos de los docentes que solo imparten su especialidad.

\section{c) Metodología y procedimiento}

La metodología desarrollada ha sido mixta (Christ, 2007), siendo un "tipo de estudio donde el investigador mezcla o combina técnicas de investigación, métodos, enfoques, conceptos o lenguaje cuantitativo o cualitativo en un solo estudio" (Johnson y Onwuegbuzie, 2004, 17). Para ello, se han utilizado cuestionarios, entrevistas semi-estructuradas, observaciones, informes escritos realizados por el grupo experimental y encuentros grupales (de los que se incluye las formaciones descritas y grupos de reflexión). En gran medida para la elaboración de este trabajo, hemos rescatado la información de las entrevistas, la observación y la formación desarrollada en el grupo experimental.

En relación a la utilización de los cuestionarios, sirvieron como un instrumento de la fase diagnostica, y fueron respondidos por la totalidad de los docentes participantes en el proyecto. Combina ítems de testeo tales como edad, sexo, años en la profesión, tiempo en el centro educativo, número de estudiantes, especialidad..., con ítems en formato de Escala Likert, incluyendo enunciados del tipo: el alumnado con necesidades específicas de apoyo requieren ser atendidos fuera del aula, la diversidad es un elemento clave para la enseñanza, me preocupa las agrupaciones dentro del aula, investigo sobre otras formas didácticas, me siento a gusto con los resultados de mi práctica docente... Cabe mencionar que, debido al objeto de este artículo, no se ha recurrido a los datos ofrecidos por los cuestionarios, tan solo algunos elementos estadísticos como el sexo o la especialidad.

Sobre las entrevistas podemos decir que se han realizado 12 entrevistas semiestructuradas a docentes de Educación Infantil y 25 a docentes de Educación Primaria, entre los cuales 18 (72\%) han sido tutores y especialistas, y $7(28 \%)$ docentes especialistas. Los participantes han respondido de un modo general a la pregunta: ¿Qué me puede contar acerca de los agrupamientos de aula? Otras preguntas frecuentes han sido: ¿Realiza actividades de aula que permitan a su alumnado agruparse de un modo diferente al habitual?; cha cambiado a lo largo del tiempo su organización de las mesas del aula?; si su respuesta es afirmativa, ¿’a qué se ha debido esta modificación?; ¿cree usted que la organización y disposición de las mesas influye en el proceso de enseñanza-aprendizaje?, con el objeto de ampliar la información aportada por los docentes.

Una vez finalizadas todas las entrevistas, dio comienzo la formación en el grupo experimental, esta se ha realizado durante el primer y segundo trimestre del curso escolar 2016/17, para posteriormente desarrollar las actividades propuestas por los docentes en el tercer trimestre.

La observación del grupo experimental ha sido realizada por el coordinador del proyecto de innovación -maestro de Pedagogía Terapéutica del centro-, el cual ha trabajado en inclusión con su alumnado a lo largo de todo el curso en dicho centro. Este hecho le ha permitido, primero, participar de un modo directo con su alumnado dentro del aula de las actividades propuestas por los docentes y, segundo, constatar a través de la observación el uso que los docentes han hecho de estas metodologías en sus aulas. En el grupo de control, ha sido realizada por los miembros del 
equipo investigador.

Al finalizar el proyecto -tercer trimestre- cada docente ha realizado un informe personal con una valoración final de la formación y la aplicación de recursos, en el que ha reflejado entre otros aspectos, las actividades que han desarrollado, las potencialidades y debilidades de las mismas, sugerencias de cambio..., a este informe se le ha sumado dos reuniones o grupos de reflexión realizados, las cuales han permitido recabar, discutir y validar la información aportada.

\section{Resultados y discusión}

\subsection{Comparación de agrupamientos y disposición en el aula}

Los resultados que presentamos centrados en los modos de agrupar al alumnado y la disposición del aula han sido utilizados en los grupos de reflexión con los docentes para analizarlos desde el prisma de la educación inclusiva. Sobre esto último, aún estamos en proceso de análisis, por lo que solo en las conclusiones plantearemos los primeros elementos abordados al respecto.

A continuación, detallamos los hallazgos diferenciando el nivel educativo (infantil o primaria) y distinguiendo entre los grupos de control y experimental.

\section{a) Grupo Control Infantil}

De forma unánime y generalizada los datos que nos han aportado las docentes que forman parte del grupo control en relación con la organización de las mesas de sus aulas han sido los siguientes.

Desde hace algunos años, de acuerdo a las entrevistas y la observación, son muchos los docentes de educación infantil que siguen una línea progresiva en relación a las agrupaciones. En el aula de infantil de 3 años la agrupación característica que usan las dos maestras suele ser en gran grupo, lo que facilita la labor del docente para la supervisión, pues este alumnado presenta unos niveles bajos de autonomía. En muy raras ocasiones trabajan en pequeños grupos y nunca lo hacen en pareja o de manera individualizada.

En el aula de infantil de 4 años por norma general el alumnado está agrupado en pequeños grupos, podemos encontrar tres o cuatro mesas dispuestas a lo largo de la clase. Estos pequeños grupos suelen ser heterogéneos, las maestras se encargan de agrupar al alumnado de tal forma que siempre estén sentados juntos alumnado con diferentes niveles de desempeño de la tarea, con el objeto de que el alumno o alumna que termine antes pueda ayudar al compañero. Hallamos que actividades como visionar un video o escuchar una canción en el ordenador son de los pocos momentos en los que el alumnado está agrupado en gran grupo. La maestra de inglés sí realiza dinámicas que fomentan el trabajo en gran grupo, estas permiten que el alumnado realice juegos cooperativos en gran grupo, pequeños grupos o parejas, es así la única docente que hemos encontrado que de forma consistente aplica una metodología de enseñanza-aprendizaje que se asienta bajo los principios del "aula inteligente", esto es, incorpora las nuevas tecnologías como instrumentos docente y discente.

En el aula de infantil de 5 años encontramos principalmente dos tipos de disposición. En uno de los casos se utiliza la organización en pequeños grupos y la otra maestra utiliza la formación en forma de U. Independientemente del tipo de organización de las mesas, en todos los casos y siempre que se pueda el alumnado está dispuesto según género de forma alternativa y teniendo en cuenta el nivel de desarrollo, con el objeto de crear grupos heterogéneos. En todos los casos las actividades puntuales, como por ejemplo la hora de la asamblea, el visionado de un video o la asignatura de inglés, las agrupaciones son diferentes, principalmente en gran grupo. Hemos de tener en cuenta que con el alumnado de esta etapa también se trabaja con rincones lo que permite que la organización del aula sea más flexible en función de la actividad que se va a desarrollar.

Entre los docentes de Educación infantil del grupo control encontramos una organización de las 
mesas del aula que queda configurada de la siguiente forma:

Figura 1

Organización de las mesas en el grupo de control de educación infantil

Agrupamientos Educación Infantil grupo control

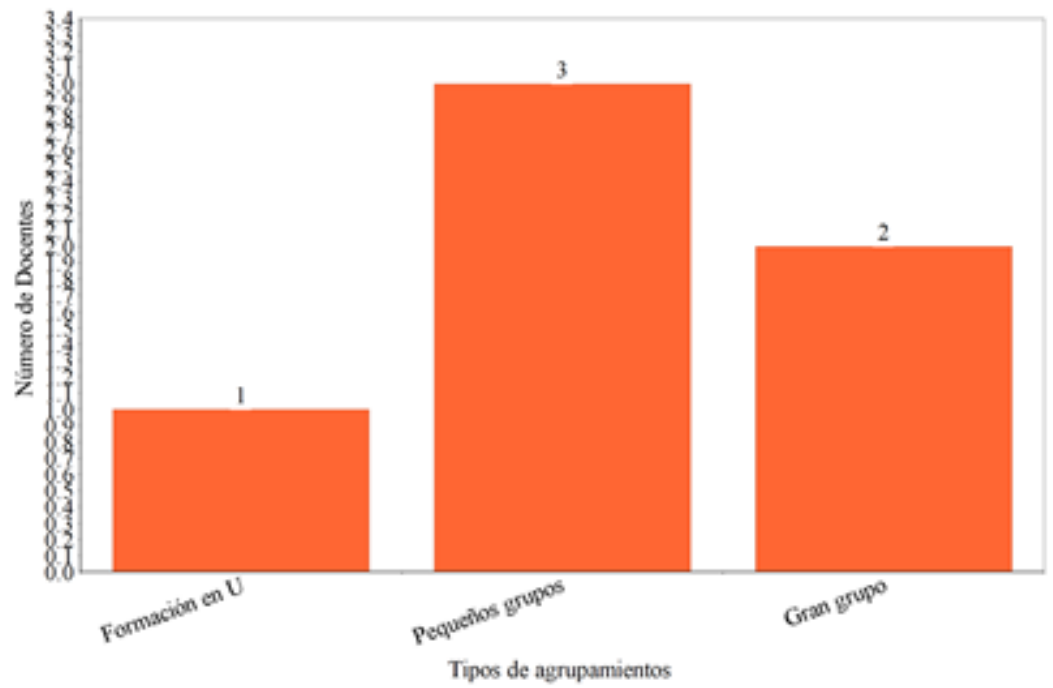

Fuente: elaboración propia.

\section{b) Grupo Experimental Infantil}

Antes de la implementación del proyecto, se ha desarrollado una etapa diagnóstica en la cual se ha observado la estructuración de las aulas de educación infantil del centro que ha actuado como grupo focal o experimental.

Los datos obtenidos de la observación muestran un patrón similar al obtenido en el grupo control. Una de las maestras del grupo de 3 años utiliza la modalidad de gran grupo, la otra maestra del otro grupo de 3 años, junto a las dos maestras de 4 años y la otra maestra de 5 años utilizan de manera consistente a lo largo de todo el curso los pequeños agrupamientos, salvo en actividades puntuales. A excepción de una de las docentes (interinas) del grupo de infantil de 5 años, la cual desarrolla en su aula a diario algunas actividades dinámicas que promueven el trabajo en diferentes espacios y agrupamientos. De estas actividades se ha servido el propio proyecto para implementarlas en las otras aulas de educación infantil; aun así, la etapa diagnóstica nos muestra el siguiente patrón.

Las actividades que se han desarrollado dentro del proyecto han conseguido que el grupo experimental utilice diferentes agrupaciones a lo largo de la semana. Estas han sido implementadas en la dinámica del aula y han supuesto una ruptura con la típica formación rígida que cada maestra utilizaba con su alumnado, y que solo se modificaba en actividades puntuales. En el tercer trimestre se ha apreciado la inclusión de actividades inclusivas y en gran grupo en dos de las aulas (una de 3 años y una de 5 años) pero de forma puntual en días esporádicos. 
Figura 2

Organización del alumnado en el grupo experimental de educación infantil

Agrupamientos Educación Infantil grupo experimental

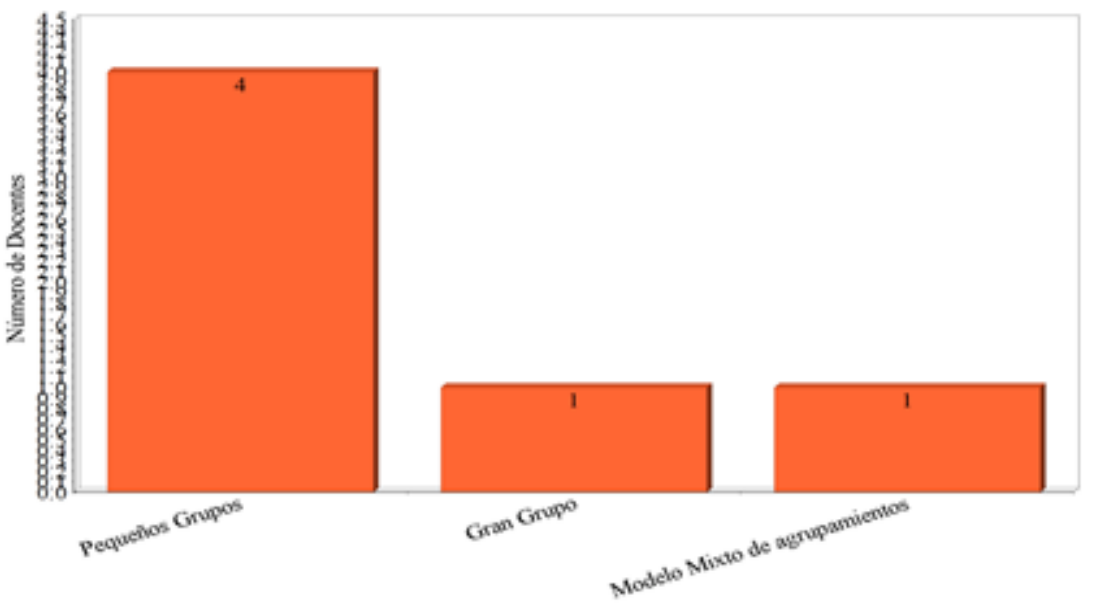

Fuente: elaboración propia.

\section{c) Grupo Control Primaria}

Las entrevistas realizadas a los docentes que forman parte del grupo control en el ámbito de la educación primaria nos reportan los siguientes resultados.

La disposición más habitual entre los tutores de las clases de primaria ha sido la formación en U, pues 3 tutores utilizan esta disposición. En actividades puntuales (trabajos, investigaciones o actividades TIC) los tutores permiten al alumnado trabajar en pequeños grupos, y ocho de los docentes consultados modifican las posiciones del alumnado al menos una vez al trimestre con el objeto de que se relacionen con otros compañeros de clase, pero no modifican el agrupamiento y/o disposición de las mesas.

De un modo más específico, encontramos a otros docentes que optan por otros tipos de agrupamiento. Entre ellos encontramos a una de las tutoras de $6^{\circ}$ de primaria (especialista de música) y a un maestro de $4^{\circ}$ de primaria (tutor) que disponen a su alumnado de forma individual como norma y en pocas ocasiones realizan actividades en pequeño o gran grupo; ambos justifican esta decisión amparándose en que su "alumnado no sabe trabajar en grupo y que hablan mucho".

Encontramos también a otro tutor de $4^{\circ}$ de primaria (especialista en educación física) el cual utiliza el agrupamiento por parejas de forma sistemática, principalmente para "fomentar la coeducación y la formación de parejas heterogéneas". Según expone "cada dos semanas modifico la organización de la clase", cambia algunas parejas y modifica su situación en el espacio; según este docente, esta opción es la que mejor le ha funcionado a lo largo de toda su trayectoria profesional.

Un tutor de $5^{\circ}$ y una tutora de $6^{\circ}$ de primaria (maestra de música) desarrollan actividades de investigación, debate, o creación de material que requieren de modificar la organización de las mesas y del aula. Estas actividades dinámicas se implementan dentro del currículum de la asignatura de Lengua Castellana y Literatura, Educación Artística, ciencias de la Naturaleza y Ciencias Sociales.

Los tres especialistas en lengua extranjera (un maestro y una maestra de inglés y una maestra de francés) prefieren una disposición en parejas agrupando al alumnado con diferentes niveles por parejas y a ser posible teniendo en cuenta la coeducación. Esta disposición les permite trabajar diálogos, "listening" o traducciones; las clases en las que se trabajan los idiomas son más dinámicas al utilizar audios y vídeos para desarrollar la asignatura, lo que facilita la motivación del alumnado y el andamiaje. En esta línea y como manifiestan los docentes, las clases de Educación Física y 
Educación Musical también permiten otro tipo de agrupaciones al desarrollar diferentes dinámicas en las que el alumnado utiliza el cuerpo, la voz y el movimiento.

Figura 3

Agrupamientos en el grupo control de educación primaria

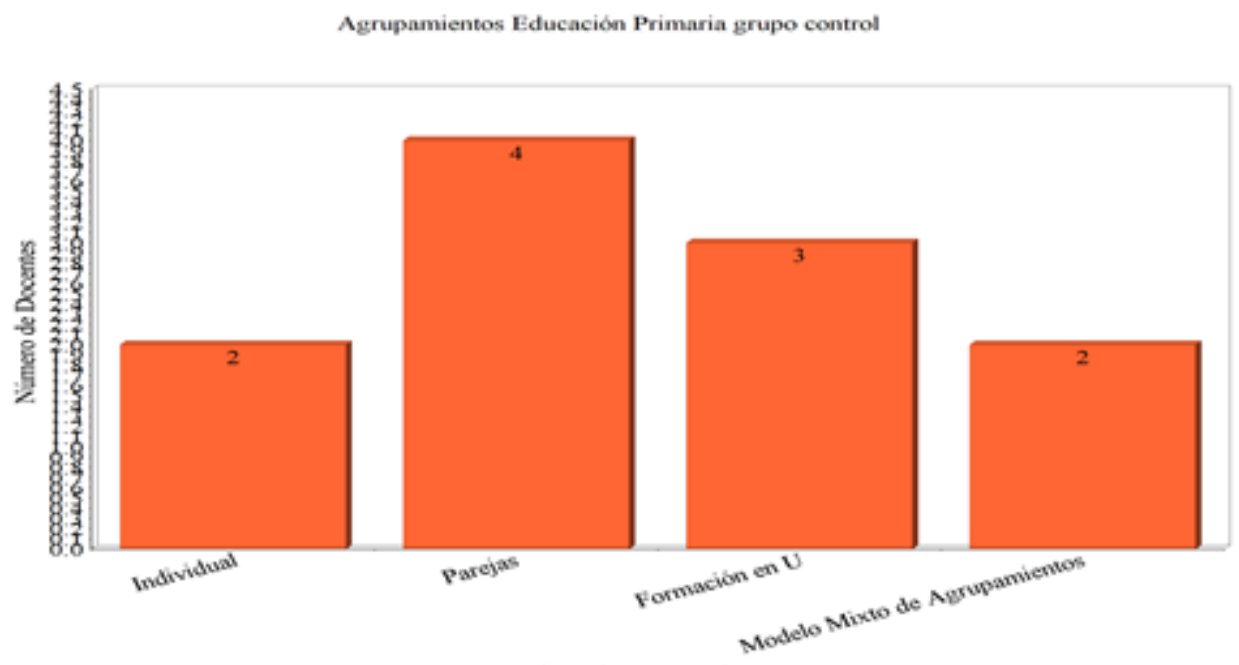

Fuente: elaboración propia.

\section{d) Grupo Experimental Primaria}

Las entrevistas a los docentes que pertenecen al centro que ha actuado como grupo experimental nos han narrado una situación diferente a la referida anteriormente. La opción más utilizada por los tutores ha sido disponer al alumnado individualmente, excepto en un curso de quinto de primaria y en las diferentes especialidades, pues estos docentes modifican los agrupamientos de forma temporal y específica para su asignatura. Por este motivo se hace necesario mostrar dos cuadros, uno en el que aparecen los agrupamientos que desarrollan los tutores en sus aulas y otro en el que se muestran los agrupamientos de los especialistas.

Los tres especialistas de Lengua extranjera e inglés, la maestra de música y el maestro de Educación Física si han utilizados diferentes disposiciones, principalmente agrupamientos en pareja en las asignaturas de lenguas extranjeras. Por su parte, los especialistas de Música y Educación Física sí utilizan el gran grupo para el desarrollo de sus materias específicas, pero en sus respectivas tutorías agrupan a su alumnado de manera individual. Del mismo modo que los especialistas en Lengua extranjera (inglés o francés) utilizan agrupamientos individualizados en sus respectivas tutorías.

Una de las tutoras de quinto de primaria (maestra de inglés) utiliza en las áreas troncales en las que imparte clases diferentes agrupamientos, según la actividad y los objetivos que se proponga. Principalmente agrupa al alumnado por parejas, pero todas las semanas plantea actividades dinámicas en pequeños grupos.

Tras las actividades formativas desarrolladas los cambios introducidos han sido coyunturales, es decir, los docentes han aplicado en sus aulas tanto las actividades planteadas en la formación, como las creadas por ellos a partir de la misma, pero no se ha apreciado en ningún docente un cambio en el agrupamiento de su alumnado y/o en la implementación de otro tipo de actividades que requieran de otra forma de agrupar. 
Agrupamientos Tuloes Educacoion Primaria grupo experimental

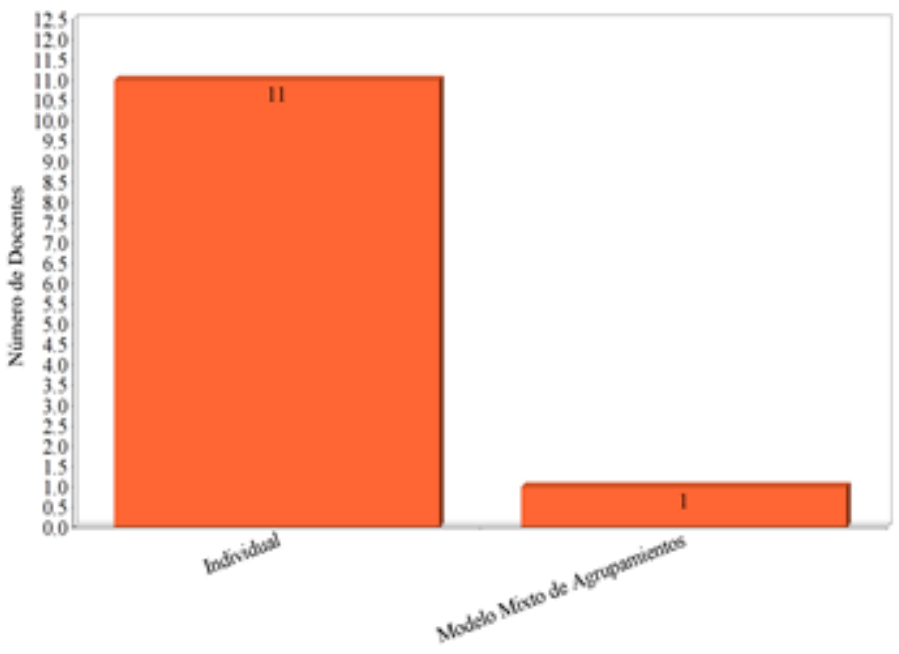

Fuente: elaboración propia.

Figura 5

Agrupamientos del profesorado especialista en el grupo experimental de educación primaria

Agrugamionios Maetros Expeciulistas Educasion Pimaria grupo experimental

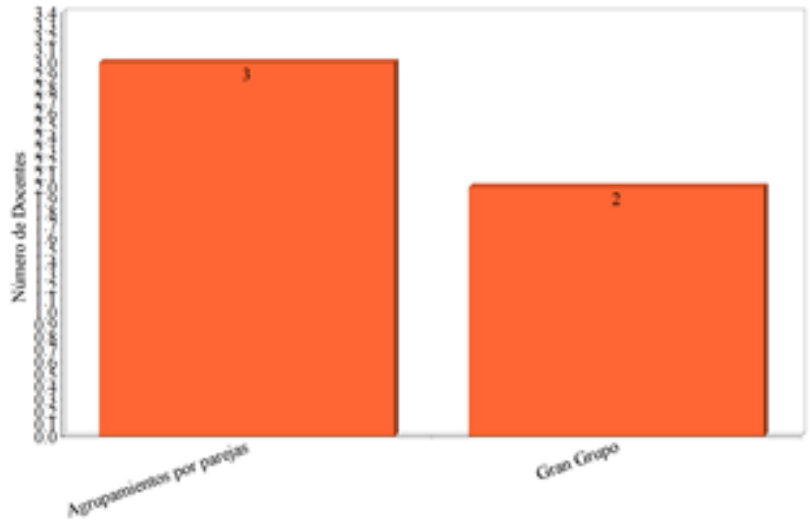

Fuente: elaboración propia.

\subsection{La valoración del proyecto de innovación}

La implantación del proyecto de innovación educativa ha supuesto, de acuerdo a las entrevistas y los primeros resultados de los encuentros grupales, una experiencia que, al menos, les haga plantearse otras formas posibles de trabajar, aunque manifiestan que requieren de mayor práctica y asesoramiento al respecto. Las categorías analíticas que emergen de las mismas las podemos incluir en tres grupos: (1) particularidades entre infantil y primaria, (2) relaciones entre la flexibilización curricular, edad del alumnado y las asignaturas, (3) agrupamientos y disposición del alumnado en el aula.

En relación a los agrupamientos y las particularidades del nivel educativo, el cambio más notable se ha producido en la etapa de educación infantil, en el que el proyecto ha supuesto un cambio metodológico prolongado para las maestras. La flexibilización curricular, la edad del alumnado y el tipo de actividades que se desarrollan en la etapa de Educación Infantil han permitido a las maestras implantar en sus aulas todas las actividades propuestas en el citado proyecto, logrando cambios significativos en las competencias y desarrollo social y/o emocional del alumnado. Todo esto se lo expresan las maestras en el encuentro grupal, añadiendo que han mejorado las relaciones sociales y 
el alumnado se ha vuelto más sociable, "el proyecto ha supuesto una oportunidad para las docentes para incorporar a sus rutinas de aula otros tipos de agrupamientos". Una vez finalizado el curso y como evaluación del proyecto, las maestras de educación infantil han valorado como positivas las actividades desarrolladas, no solo en el plano conceptual, también en el plano social y cooperativo, el cual se ha desarrollado a partir del uso de diferentes espacios y agrupamientos escolares, tal y como pretendía el citado proyecto.

En contraposición, los docentes de Educación Primaria que han participado en el proyecto han aplicado algunas actividades que han requerido que su alumnado trabaje en parejas, en pequeños y grandes grupos, y han sido conscientes de las ventajas que reporta en su alumnado la utilización de diferentes agrupamientos para trabajar. Aun así, y a excepción de uno de los docentes (tutora de $5^{\circ}$ de primaria), solo se han utilizado diferentes agrupamientos escolares en momentos puntuales, cuando se han aplicado las actividades directamente relacionadas con el proyecto.

Los resultados que se han obtenido con los docentes de Primaria en relación al uso de agrupamientos escolares diversos han sido muy distintos. De forma generalizada estos han mantenido una organización escolar individualizada, a excepción de las actividades que se han implementado por estar relacionadas con el proyecto, así como por parte de uno de los docentes (tutora de $5^{\circ}$ de primaria) que ha incorporado a su rutina de aula actividades de debate, trabajo en equipo e investigación en las materias de Lengua Castellana y Literatura, Ciencias naturales y Ciencias Sociales, las cuales según sus palabras "han hecho que mis alumnos se expresen en voz alta y den su opinión".

Se ha podido comprobar que la utilización de diferentes agrupamientos escolares ha sido un aspecto coyuntural en las aulas de Primaria, estos se han producido porque todas las actividades del proyecto requieren de modificaciones temporales en las agrupaciones.

Los especialistas en lengua extranjera (inglés y francés) prefieren una disposición en parejas agrupando al alumnado con diferentes niveles por parejas y a ser posible teniendo en cuenta la coeducación. Esta disposición les permite trabajar diálogos, "listening" o traducciones. Las clases en las que se trabajan los idiomas son más dinámicas al utilizar audios y vídeos para desarrollar la asignatura, lo que facilita la motivación del alumnado y el andamiaje de conceptos. En esta línea las clases de Educación Física y Educación musical también permiten otro tipo de agrupaciones al desarrollar diferentes dinámicas en las que el alumnado utiliza el cuerpo, la voz y el movimiento.

\section{Conclusiones}

El aula, como hemos expresado, es un espacio de encuentro y de trabajo entre el alumnado y en la que el docente, en una primera instancia, se asienta como guía que promueve el aprendizaje; la organización del aula queda supeditada al modelo pedagógico que el docente o equipo educativo persiga en su proyecto de centro y de las relaciones docente-alumnado y alumnado-alumnado que se generan del mismo.

Esto se ha hecho plausible en los resultados expuestos anteriormente. Aún en experiencias puntuales, como la aplicación de estrategias fruto de la formación realizada, se ha observado cómo el alumnado ha manifestado cambios a la hora de relacionarse y de sentirse en el aula, mostrando, según las observaciones realizadas y los encuentros grupales, mayor interés y ganas. Este hecho también se ha constatado, como decíamos párrafos atrás, en materias de idiomas o artísticas.

Esto nos hace plantearnos que precisamente en esa discusión entre lo organizativo y lo metodológico, que en el marco teórico expresábamos, residen fuertes posibilidades que requieren de una forma más interactiva de enfocar la enseñanza. Las materias de idiomas o educación artística, por ejemplo, vienen per se dispuestas a otros lenguajes, lo interesante sería extrapolarlo a otras disciplinas del currículum escolar, incluso, desbordar la visión fragmentada del mismo.

En referencia a la organización del aula, podemos afirmar que no es lo mismo que el profesorado 
sea el eje de atención en el aula o que, por el contrario, el alumnado sea el que participe en la concepción del currículum. Algunos ejemplos lo hemos observado en algunas asambleas en las que se ha debatido sobre una película; hemos denotado un alto potencial curricular generado por el alumnado, debido a que se sienten libres de expresar, pensar, construir... Son sus inquietudes las que se ponen sobre la mesa. El mayor problema reside, que este tipo de actividades y organización pasan a ser anecdóticas (o sin valor atribuido en procesos de evaluación) y no se integran como práctica educativa cotidiana.

Y es a partir de aquí que nos estamos planteando en los grupos de reflexión con el profesorado participante en el proyecto, de qué manera se pueden trazar otras formas de agrupar (que condicionen cambios metodológicos) para, por un lado, generar prácticas educativas inclusivas y, por otro lado, generar aprendizajes de calado en el alumnado. Este interés no solo viene del equipo investigador, sino que en la fase de reflexión compartida encontramos dos categorías emergentes que están apareciendo con fuerza: inclusión y clima escolar.

De la aplicación del proyecto de innovación se desprenden varios aspectos que están apareciendo en el análisis de la información aportada de los grupos de reflexión:

- La plasticidad que muestra el currículum de la educación infantil se ha visibilizado tanto en la facilidad para incorporar actividades dinámicas al currículum, como en la capacidad del alumnado para adaptarse a cualquier tipo de organización espacial del aula.

- Que la capacidad de trabajo en equipo, argumentación, respeto mutuo o capacidad de debate del alumnado es superior a la esperada por parte de sus docentes.

- Una organización inteligente del aula permite crear espacios y tiempos educativos en los que el alumnado desarrolle la expresión oral, actitud de respeto y espíritu crítico.

- La obsesión que tienen los docentes de educación primaria por impartir todo el currículum, les incapacita para desarrollar actividades en las que usen diferentes agrupamientos, adoleciendo falta de tiempo para ello.

- En materias como Educación Musical, Educación Física o Lengua extranjera (Inglés o Francés) los docentes que comparten especialidades y tutorías utilizan diferentes agrupamientos escolares en sus respectivas especialidades que después no utilizan en las asignaturas que imparten como tutores (Lengua Castellana y Literatura, Matemáticas, Ciencias Naturales y Ciencias Sociales).

Todos estos aspectos corroboran la dificultad a la que nos enfrentamos al intentar modificar cualquier metodología de aula en relación con los agrupamientos. Por regla general los docentes se sienten cómodos aplicando las actividades en el modo, tiempo y agrupamientos en los que siempre lo han hecho, esto es, de acuerdo a las tradiciones pedagógicas en las que se han formado o involucrados profesionalmente.

En este punto, nos formulamos una serie de cuestiones en forma de retos que nos planteamos diariamente a la hora de enfrentar y emprender nuestra práctica docente y que se están trabajando en la fase reflexiva y propositiva con los participantes. Por un lado, algunas referidas al plano de la organización escolar: ¿qué de educativo-pedagógico tienen nuestras decisiones curriculares, organizativas y metodológicas?, ¿de qué manera lo educativo está íntimamente ligado a la participación y la democracia?, ¿se aprende a través de la participación y la inclusión?, ¿cómo atender de manera inclusiva al triángulo esencial de cualquier práctica educativa, esto es, currículo, metodología, aprendizaje?

Por otro lado, otras preguntas-reto, aunque relacionadas con lo anterior, centradas en la inclusión educativa y rescatada de Pujolàs:

“¿Cómo pueden progresar en su aprendizaje alumnos "diferentes" a la mayoría de los que asisten a una escuela (diferentes por varios motivos: porque tienen alguna discapacidad, porque son de culturas distintas y no dominan la lengua predominante, porque pertenecen a un entorno social marginado...) en un aula en la cual cada uno trabaja solo en su pupitre y en la cual el profesor o la profesora debe 
atender individualmente a sus estudiantes tan "diversos" unos de otros?; ¿cómo pueden progresar estos alumnos "diferentes" en un aula en la cual los estudiantes compiten entre ellos para lograr ser el primero, el mejor, sea como sea?” (2009, p. 5)

En este sentido, y de acuerdocon los registros y resultados de este estudio, pensamos que gran parte de las prácticas escolares están basadas en una lógica eficientista y tecnocrática en las que se intenta fragmentar conocimiento, tiempo (Palau y Cela, 1997), espacios, etc. Todo ello con el objetivo de conseguir, de manera homogénea y universal, que el alumnado acceda a unas bases culturales y cognitivas adecuadas para la sociedad actual (Cortés, Leite y Rivas, en prensa). Esta lógica está anclada en la construcción moderna de escuela y choca de frente precisamente con "aquellas formas, modos, contenidos y empaque de las nuevas representaciones societarias actuales; y ya no solo por los avances tecnológicos, sino además por los progresos en materia de educación, psicología, neurología...” (opus cit.).

Para fomentar otra forma de disponer y establecer agrupamientos en el aula escolar, pensamos que no es simplemente una cuestión de escenarios o espacios de aprendizajes, sino que desborda y se inserta al ámbito de la relación humana que predispone al sujeto al aprendizaje; esto es, los espacios educativos limitan o multiplican las posibilidades y las potencialidades educativas precisamente por la disposición que tengan los discentes y docentes de experimentar formas de construcción del conocimiento sujeta al diálogo, a la controversia, a la discusión... En otras palabras, una forma sujeta a la cooperación y a la inclusión (Pujolàs, opus cit.).

\section{Referencias}

Ainscow, M., Booth, T., \& Dyson, A. (2006). Improving Schools, Developing Inclusion. Nueva York: Routledge.

Ball, S.J. (1990). Foucault and education: Disciplines and knowledge. Londres: Routledge.

Barrera, A.G. (2013). Proponiendo un concepto nuclear latente en educación: las Necesidades Educativas Personales (NEP). (Tesis inédita de Doctorado). Universidad Autónoma de Madrid, Repositorio UAM.

Blase, J. (2002). Las micropolíticas del cambio educativo. Profesorado, Revista del Profesorado y currículum, 6, 1-2. Recuperado el 10 de febrero de 2018 de: https://www.ugr.es/ recfpro/rev61ART2.pdf

Casanova, M.A. (2011). Educación inclusiva: un modelo de futuro. Madrid: Wolters Kluwer.

Christ, T. (2007). A Recursive Approach to Mixed Methods Research in a Longitudinal Study of Postsecondary Education Disability Support Services. Journal of Mixed Methods Research, 1(3), pp. 226-241. doi: 10.1177/1558689807301101

Cortés, P, Leite, A., \& Rivas, J.I. (en prensa). Relatos con corazón. Aprendizaje con estudiantes universitarios a través de sus autobiografías. En Padua, D., Márquez, M.J., \& Prados, E. (Eds.). Educar con corazón. Almería: Universidad de Almería.

Cortés, P., \& Jiménez, A. (2016). Identidades resilientes, identidades creativas. ¿'Acaso la botella es siempre verde? In Cortés, P., \& Márquez, M.J. (Eds.). Creatividad, comunicación y educación. Más allá de las fronteras del saber establecido (pp. 35-50). Málaga: UMA Editorial.

Cortés, P., Leite, A., \& Rivas, J.I. (2014). Un enfoque narrativo de la identidad profesional en profesorado novel. Tendencias Pedagógicas, 24, pp. 199-214. Recuperado de: https://revistas.uam.es/tendenciaspedagogicas/article/view/2101/2199.

Delgado, W. (2007). Inclusión: principio de calidad educativa desde la perspectiva del desarrollo humano. Revista Educación, 31(2), pp. 45-58. doi: 10.15517/revedu.v31i2.1243

Dyson, A. (2010). Changes in special education theory from an English perspective. Revista Internacional de Educación Inclusiva, 3(1), pp. 69-84.

Gardner, H. (1983). Estructuras de la mente: la teoría de las Inteligencias Múltiples. Colombia: Fondo de Cultura Económica.

Goleman, D. (1996). Inteligencia emocional. Barcelona: Kairós.

Guasp, J.J.M., Ramón, M.R.R., \& De la Iglesia Mayol, B. (2016). Buenas prácticas en educación inclusiva. Educatio Siglo XXI, 34, pp. 31-50. doi: 10.6018/j/252521.

Higgins, S. (2005): The impact of school environments: a literature review. The Centre for Learning and Teaching School of Education, Communication and Language Science. University of Newcastle. Recuperado el 9 
de febrero de 2018 de: http://www.academia.edu/446530/The_Impact_of_School_Environments_A_Literature_Revi ew.

Johnson, B., \& Onwuegbuzie, A. (2004). Mixed Methods Research: A Research Paradigm Whose Time Has Come. Educational Researcher, 33(7), pp. 14-26. doi: 10.3102/0013189X033007014.

Laorden Gutiérrez, C., \& Pérez López, C. (2002). El espacio como elemento facilitador del aprendizaje. Una experiencia en la formación inicial del profesorado. Pulso: Revista de Educación, 25, pp. 133-146.

Morán, C, Molina, S., \& Siles, G (2012). Aportaciones científicas a las formas de agrupación del alumnado. Revista de Organización y Gestión Educativa, 2, pp. 13-18.

Murillo Torrecilla, F. J., Martínez Garrido, C. A., \& Hernández Castilla, R. (2011). Decálogo para una enseñanza eficaz. REICE. Revista Iberoamericana sobre Calidad, Eficacia y Cambio en Educación, 9(1), pp. 7-27.

Palau, J., \& Cela, J. (1997). El espacio. Cuadernos de Pedagogía, 254, pp. 68-70.

Pujolàs, P. (2009). Aprendizaje cooperativo y educación inclusiva: una forma práctica de aprender juntos alumnos diferentes. VI Jornadas de cooperación educativa con Iberoamérica sobre educación especial e inclusión educativa. Guatemala. Recuperado el 23 de enero de 2018 de: https://www.mecd.gob.es/dms-static/f4d240d3-55ad-474f-abd7-dca54643c925/2009ponencia-jornadas-antiguas-pere-pdf.pdf.

Quintana Aparicio. E. (2003). Estudio del mobiliario escolar y su influencia en la postura sedente en una población infantil. Departamento de Física, ingeniería y radiología médica. Área de fisioterapia. E.U de Enfermería y fisioterapia. Universidad de Salamanca.

Rivas, J.I. (2003). La perspectiva cultural de la organización escolar: marco institucional y comportamiento individual. Educar, 31, pp. 109-119. doi: 10.5565/rev/educar.307.

Rivas, J.I., Leite, A., Cortés, P., Márquez, M.J., \& Padua, D. (2010). La configuración de identidades en la experiencia escolar. Escenarios, sujetos y regulaciones. Revista de Educación, 353, 187-209.

Rojas Tejada, A. J., Sayans-Jiménez, P., Cruz del Pino, R. M., \& Tatar, M. (2012). Creencias del Profesorado de Español sobre los agrupamientos inclusivos del programa de Aulas Temporales de Adaptación Lingüística. Electronic Journal of Research in Educational Psychology, 10(28), 1249-1266.

Ruiz Ruiz, J.M. (1994). Espacio Escolar. Revista Complutense de Educación, 5(2), pp. 93-104.

Segovia Olmo, F. (2003). El aula inteligente. Nuevas perspectivas. Madrid: Espasa- Calpe.

Segovia, F., \& Beltrán, J. (1998). El aula inteligente, nuevo horizonte educativo. Madrid: Editorial Calpe.

Terrén, E. (2004). Las organizaciones educativas como sistemas de comunicación. Revista Iberoamericana de Educación, 36, pp. 189-214. Recuperado el 7 de febrero de 2018 de: http://rieoei.org/rie36a10.htm.

Trilla, J., \& Puig, J. M. (2003). El aula como espacio educativo. Cuadernos de Pedagogía, 325, pp. 52-55.

Visedo J.M. (1991). Espacio escolar y reforma de la enseñanza. Revista Interuniversitaria de Formación del Profesorado, 11, pp. 125-135. 